Revista General de Información y Documentación ISSN: 1132-1873

http://dx.doi.org/10.5209/RGID.60806

\title{
Estudio webmétrico de los Archivos Nacionales Iberoamericanos: evaluación heurística vs evaluación automática
}

\author{
Isabel María Sanz Caballeroㅜㅜ ${ }^{1}$ Cristina Faba Pérez ${ }^{2}$
}

Recibido: 21 de noviembre 2017 / Aceptado: 22 de abril de 2018

Resumen. El propósito del presente trabajo es doble: por un lado, obtener los rankings de calidad de los sitios web de los Archivos Nacionales en Iberoamérica (España, Portugal y Latinoamérica), tras la implementación de modelos ponderados de características subjetivas generales y específicas (modelos heurísticos) y automáticas (modelos automáticos) diseñados en la presente investigación, y por otro, comprobar si existe correlación entre dichos rankings y diversos indicadores del país donde se ubica el Archivo, así como entre los propios rankings hallados mediante la evaluación heurística y la evaluación automática. Para ello, se diseña el modelo ponderado de características que permite la evaluación específica de los sitios web de archivos, además de una plantilla exhaustiva de características para realizar ambas evaluaciones. Los resultados muestran un mayor cumplimiento en el caso de las características generales y automáticas; una relación sustancial y moderada directa entre el ranking heurístico y los indicadores del país, e inversa entre el ranking automático y dichos indicadores; y una correlación moderada inversa entre el ranking heurístico y automático. Los resultados permiten concluir que es necesario realizar sitios web más orientados al Archivo y combinar ambos tipos de evaluaciones para obtener resultados más certeros.

Palabras Clave: Archivos Nacionales; Iberoamérica; Sitios web; Ranking; Evaluación.

\section{[en] Webmetric study of Ibero-america National Archives: Heuristic evaluation vs Automatic evaluation}

\begin{abstract}
The purpose of this paper is twofold: to obtain the Quality rankings of the National Archives websites in Ibero-America (Spain, Portugal and Latin America), following the implementation of weighted models of general and specific subjective characteristics (Heuristic models ) and automatic (Automatic models) models designed in the present investigation, and on the other, to verify if there is a correlation between these Rankings and different indicators of the country where the Archive is located, as well as between the Rankings found through the Heuristic evaluation and the Automatic evaluation. To do this, we designed the weighted model of characteristics that allows the specific evaluation of the archival web sites, in addition to an exhaustive template of characteristics to perform both evaluations. The results show greater compliance in the case of the general and automatic characteristics, a substantial and moderate direct relationship between the Heuristic ranking and the country's indicators, and the reverse between the Automatic ranking and these indicators; And a moderate inverse correlation between the Heuristic and Automatic ranking,
\end{abstract}

1 Universidad de Extremadura. Departamento de Información y Documentación

E-mail: isancab@gmail.com

2 Universidad de Extremadura. Departamento de Información y Documentación

E-mail: cfabper@unex.es 
which allows to conclude that it is necessary to design websites that are more archives oriented and to combine both types of valuations to obtain more accurate results.

Keywords: National Archives; Ibero-america; Websites; Ranking; Evaluation.

Sumario. 1. Introducción. 2. Datos y metodología. 3. Resultados y discusión. 4. Conclusiones. 5. Referencias bibliográficas

Cómo citar: Sanz Caballero, I. M.; Faba Pérez, C. (2018) Estudio webmétrico de los Archivos Nacionales Iberoamericanos: evaluación heurística vs evaluación automática, en Revista General de Información y Documentación 28 (1), 23-44.

\section{Introducción}

Es evidente que la aplicación de las nuevas tecnologías en los archivos ha permitido que el usuario pueda acceder a determinada información y a los servicios que ofrecen con mayor rapidez. Sin embargo, en general, aunque facilitan la búsqueda de información, están aún muy lejos de disponer de herramientas que permitan al usuario consultar los documentos eficazmente (Acuña y Agenjo, 2005). Para Fuster (1999), el rescate de la información conservada en los archivos interesa, primordialmente, a las entidades productoras de los documentos, a la Administración y a las empresas, pero también a la sociedad en general, por cuanto la gestión de esta información conservada en los archivos facilita la toma de decisiones hasta el punto de que los que logran rescatar esta información, gestionándola de forma rápida y organizada, están en evidente ventaja sobre quienes no usan suficientemente sus archivos como fuente de información

Según Cerdá (2008), el modelo actual de archivo está en un momento de cambios profundos. El desarrollo de los archivos on-line es una realidad que se está extendiendo de modo imparable, ya que Internet evita los desplazamientos a los lugares físicos para tratar de localizar la información deseada. Pero este incremento de los archivos en la Red (Liria, 2001) también ha traído consigo la necesidad de comprobar si dichos archivos están funcionando correctamente y qué nivel de calidad presentan (Sanz-Caballero y Faba-Pérez, 2010). Para ello, es preciso evaluarlos atendiendo a unos parámetros o características relacionadas con su diseño, estructura o contenido. Estos parámetros forman parte de un conjunto de indicadores conocidos como "indicadores web(o)métricos de características" (AlDuwairi, Faba-Pérez y Vargas-Quesada, 2010).

El análisis de características consiste en identificar las características de los sitios web que mejor satisfacen las necesidades de los usuarios que navegan por ellos. Este análisis se fundamenta en el hecho de que los sitios web por más disímiles que sean, tienen unas características comunes o indicadores de calidad a los que se les puede asignar una calificación determinada. Dichas características pueden ser recopiladas, o bien por los analistas, o bien de forma automática, lo que justifica, en cierta medida, su objetividad (Faba-Pérez, Guerrero-Bote y MoyaAnegón, 2004). Este análisis de características se enmarca en la Webmetría (una disciplina muy relacionada con la Altmetría, centrada en el estudio de indicadores basados en la Web 2.0 para el análisis de la actividad académica y científica, de la 
que se han realizado investigaciones interesantes como Más-Bleda y Aguillo (2013) o Más-Bleda, et al. (2014).

Existen bastantes trabajos sobre análisis de características en el contexto de las unidades informativas. Sobre todo, se han realizado investigaciones en el campo de las bibliotecas, la mayoría centradas en el análisis de características subjetivas (Clyde, 1996; Clausen, 1999; Falk, 1999; Joint, 2001; Osorio, 2001; Chao, 2002; Clyde, 2004; Novljan y Žumer, 2004) aunque también existen algunos casos de modelos basados en indicadores formales (Stover y Zink, 1996 Craven, 2000; Spindler, 2002; Providenti, 2004; Schmetzke, 2005; Caballero-Cortés, Faba-Pérez y Moya-Anegón, 2009).

En el caso de los archivos, son escasas las investigaciones sobre análisis webmétricos de sus características, ya que, aunque se pueden encontrar trabajos que relacionan los archivos con Internet y la World Wide Web (MartínezGonzález, 2000; Gil-García, 2001, 2003 y Torrado-Morales, 2007), son exiguos los que desarrollan modelos webmétricos de indicadores o características. Algunos de estos últimos los podemos encontrar en trabajos como los de Cerdá (2002), Cuadrado y Gómez (2002), Gil (2003), Roba (2003), Crespo (2007), Kim y Lee (2007), Chaín y García (2009), Chaín y Más-Bleda (2009), Más-Bleda (2010),MásBleda y Chaín (2009), Sanz-Caballero y Faba-Pérez (2012) o Faba-Pérez y SanzCaballero (2014).

\subsection{Objetivos}

En la presente investigación, se diseña un modelo de características para la evaluación heurística y otro para la evaluación automática de Archivos Web, se implementan ambos modelos en la población de los sitios web de los Archivos Nacionales Iberoamericanos (aquellos constituidos por fondos documentales producidos por instituciones cuya jurisdicción abarca todo el territorio nacional (Diccionario de Terminología Archivística, 1995)) obteniendo respectivos rankings de calidad, y se contrasta la correlación existente, por una parte, entre dichos rankings y diversos indicadores económicos, tecnológicos, demográficos y culturales del país donde su ubica el Archivo y, por otra, entre ambos tipos de evaluaciones, siendo este último un elemento muy novedoso entre las investigaciones al respecto que parte de la siguiente hipótesis: "En general, dado que la evaluación heurística se centra en gran medida en el criterio del analista y la evaluación automática utiliza herramientas informáticas, se prevé que exista una escasa relación entre los rankings obtenidos en ambas evaluaciones”.

\subsection{Fuentes}

Para recopilar la población objeto de estudio, formada por los sitios web de los Archivos Nacionales Iberoamericanos, se ha utilizado la fuente oficial "Directorio Web de Archiveros Españoles en la Función Pública (AEFP)"33 (miembro del 
Consejo Internacional de Archivos y Asociación Latinoamericana de Archivos). La Tabla 1, ordenada por los tres ámbitos geográficosanalizados, muestra la población final de análisis formada por un total de 21 sitios web de Archivos Nacionales (1 en España, 1 en Portugal y 19 en Latinoamérica -en el resto de los países de Latinoamérica con Archivo Nacional, o bien el Archivo no disponía de sitio web o no se encontraba operativo en el momento del análisis).

Tabla 1. Población de estudio: Archivos Nacionales Iberoamericanos

\begin{tabular}{|c|c|c|c|}
\hline PAÍS & CÓDIGO & ARCHIVO NACIONAL & URL \\
\hline Portugal & $\mathrm{PO}$ & $\begin{array}{l}\text { Arquivo Nacional Torre do } \\
\text { Tombo }\end{array}$ & http://antt.dglab.gov.pt/ \\
\hline España & ES & Archivo Histórico Nacional & $\begin{array}{l}\text { http://www.mcu.es/archivos/MC/AH } \\
\text { N/ }\end{array}$ \\
\hline Argentina & AR & Archivo General de la Nación & $\begin{array}{l}\text { http://www.mininterior.gov.ar/agn/ag } \\
\text { n.php }\end{array}$ \\
\hline Bolivia & $\mathrm{BO}$ & Archivo Nacional de Bolivia & $\begin{array}{l}\text { http://www.archivoybibliotecanacion } \\
\text { ales.org.bo/ }\end{array}$ \\
\hline Brasil & BR & Arquivo Nacional de Brasil & http://www.arquivonacional.gov.br/ \\
\hline Chile & $\mathrm{CH}$ & Archivo Nacional de Chile & http://www.archivonacional.cl/ \\
\hline Colombia & $\mathrm{CO}$ & Archivo General de la Nación & http://www.archivogeneral.gov.co/ \\
\hline Costa Rica & CR & $\begin{array}{l}\text { Dirección General del Archivo } \\
\text { Nacional de Costa Rica }\end{array}$ & http://www.archivonacional.go.cr/ \\
\hline Ecuador & EC & Archivo Nacional del Ecuador & http://www.ane.gob.ec/ \\
\hline El Salvador & EL & Archivo General de la Nación & http://www.cultura.gob.sv/agn/ \\
\hline Guatemala & GU & $\begin{array}{l}\text { Archivo General de } \\
\text { Centroamérica (Guatemala) }\end{array}$ & $\begin{array}{l}\text { http://www.archivogeneraldecentroa } \\
\text { merica.com/ }\end{array}$ \\
\hline Honduras & $\mathrm{HO}$ & Archivo Nacional de Honduras & $\begin{array}{l}\text { http://www.cdihh.ihah.hn/site/index.p } \\
\text { hp/archivo-nacional }\end{array}$ \\
\hline México & $\mathrm{ME}$ & Archivo General de la Nación & http://www.agn.gob.mx/ \\
\hline Nicaragua & NI & Archivo General de la Nación & $\begin{array}{l}\text { http://www.inc.gob.ni/bibliotecas-y- } \\
\text { archivos/ }\end{array}$ \\
\hline Panamá & PA & Archivo Nacional de Panamá & http://www.archivonacional.gob.pa/ \\
\hline Paraguay & PY & Archivo Nacional de Asunción & $\begin{array}{l}\text { http://www.archivonacionaldeasuncio } \\
\text { n.org/ }\end{array}$ \\
\hline Perú & $\mathrm{PE}$ & Archivo General de la Nación & http://www.agn.gob.pe/ \\
\hline Puerto Rico & PR & Archivo General de Puerto Rico & $\begin{array}{l}\text { http://www.icp.gobierno.pr/programa } \\
\text { s/archivo-general-de-puerto-rico }\end{array}$ \\
\hline $\begin{array}{l}\text { República } \\
\text { Dominicana }\end{array}$ & $\mathrm{RD}$ & Archivo General de la Nación & http://www.agn.gov.do/ \\
\hline Uruguay & UR & Archivo General de la Nación & http://www.agn.gub.uy/ \\
\hline Venezuela & VE & Archivo General de la Nación & http://www.agn.gob.ve/ \\
\hline
\end{tabular}

Fuente: Elaboración propia (2016)

\section{Datos y metodología}

Para realizar la presente investigación, se llevan a cabo dos tipos de evaluaciones: la heurística, que requiere la participación de los analistas en la recolección manual de los datos (con la posible componente subjetiva) y la automática, más objetiva, 
ya que no precisa la participación humana en la recopilación de datos. Para este análisis automático, se usa la herramienta Linkbot 6.0, un comprobador de enlaces del mercado que tiene la capacidad de explorar de forma automática las páginas de una determinada URL extrayendo una serie de indicadores formales que consideramos importantes para el presente análisis.

Para llevar a cabo ambas evaluaciones, se diseña un modelo ponderado de características que permite la evaluación específica de los sitios web de Archivos y, por consiguiente, hallar su posicionamiento en un ranking de calidad. Dicho modelo requiere la delineación de tres parámetros:

\section{a) Diseño de plantillas de características y ponderación de las mismas}

Para idear la plantilla de evaluación heurística, se han tenido en cuenta trabajos previos sobre las características generales que debe tener un sitio web (Jiménez, 2001; Nielsen y Thair, 2002; Hassan y Martín, 2003 o Jiménez, 2007), así como sobre la naturaleza y las características específicas para evaluar sitios web archivísticas (Cerdá, 2002; Cuadrado y Gómez, 2002; Gil, 2003; Roba, 2003; Crespo, 2007; Kim y Lee, 2007; Chaín y García, 2009; Chaín y Más-Bleda, 2009;Más-Bleda y Chaín (2009); Sanz-Caballero y Faba-Pérez; 2012 o Faba-Pérez y Sanz-Caballero, 2014). En la presente investigación se han propuesto 80 características heurísticas (20 generales y 60 específicas).

Con respecto a la plantilla para la evaluación automática, se han seleccionado una serie de características ofrecidas por la herramienta Linkbot 6.0, algunas de las cuales han sido utilizadas en otras investigaciones como Faba-Pérez, GuerreroBote y Moya-Anegón (2004, 2005). Siguiendo, en parte, la metodología utilizada por dichas investigaciones, los indicadores se han agrupado en dos categorías denominadas "indicadores favorables y desfavorables", en función de si un mayor grado de cumplimiento del criterio tiene un impacto positivo o negativo, respectivamente, en la valoración del sitio web. En la presente investigación se han propuesto 10 características automáticas o formales (8 negativas o desfavorables y 2 positivas o favorables).

Una vez creadas las dos plantillas, se procede a ponderar cada una de las características en función de su importancia para evaluar una web archivística. Para ello, se cuenta con la participación de un grupo de 26 expertos en Archivística y en Webmetría, quienes valoran cada característica entre $0 ; 0,25 ; 0,5 ; 0,75$ y 1 . Tras obtener el peso de cada característica utilizando la valoración media de los expertos, la Tablas 2 muestra las plantillas diseñadas. 
Tabla 2. Evaluación heurística y evaluación automática:

Características y ponderación media

\begin{tabular}{|c|c|c|}
\hline \multicolumn{3}{|c|}{ EVALUACIÓN HEURÍSTICA } \\
\hline & CARACTERÍSTICAS GENERALES & PESOS \\
\hline C1 & Los objetivos del sitio web se expresan con claridad & 0,82 \\
\hline $\mathrm{C} 2$ & Tiene una URL correcta, clara y fácil de recordar & 0,85 \\
\hline C3 & Posee una URL estable & 0,94 \\
\hline C4 & La estructura general del sitio web está orientada al usuario & 0,92 \\
\hline C5 & Aporta un diseño general del sitio web coherente & 0,90 \\
\hline C6 & Se permite la visualización del sitio con navegadores estándar & 0,94 \\
\hline C7 & Tiene un acceso rápido y regular & 0,88 \\
\hline $\mathbf{C 8}$ & Aparece la fecha de actualización del recurso & 0,67 \\
\hline C9 & El logotipo es significativo, identificable y suficientemente visible & 0,75 \\
\hline C10 & Ofrece algún enlace con información sobre el sitio web, 'webmaster' & 0,58 \\
\hline C11 & Existe la posibilidad de convertir o traducir a otros idiomas & 0,57 \\
\hline C12 & Se cuida la gramática y la ortografía & 0,91 \\
\hline C13 & Se menciona el área geográfica que abarca & 0,77 \\
\hline C14 & 1 párrafo = 1 idea & 0,67 \\
\hline C15 & Dispone de un mapa sensible & 0,61 \\
\hline C16 & Contiene pautas de Accesibilidad & 0,73 \\
\hline C17 & Contiene rótulos significativos & 0,88 \\
\hline C18 & Funcionan los enlaces de la home page, sean internos o externos & 0,84 \\
\hline C19 & Tiene enlaces fácilmente reconocibles como tales & 0,81 \\
\hline \multirow[t]{3}{*}{$\mathbf{C 2 0}$} & No tiene sobrecarga informativa & 0,79 \\
\hline & CARACTERÍSTICAS ESPECÍFICAS & \\
\hline & INFORMACIÓN GENERAL & \\
\hline C21 & $\begin{array}{l}\text { Aparecen la misión y funciones principales del archivo, se hace mención a la } \\
\text { historia de archivo, etc }\end{array}$ & 0,93 \\
\hline \multirow[t]{2}{*}{ C22 } & $\begin{array}{l}\text { Se representa la Memoria Anual de las actividades profesionales realizadas en el } \\
\text { Archivo }\end{array}$ & 0,83 \\
\hline & VALORACIÓN: SELECCIÓN Y ELIMINACIÓN & \\
\hline $\mathrm{C} 23$ & Se refleja la tabla de valoración o calendario de conservación & 0,69 \\
\hline C24 & Existe creada una Comisión para la Valoración de documentos & 0,66 \\
\hline
\end{tabular}




\begin{tabular}{|c|c|c|}
\hline C25 & Se hace público el inventario de eliminación & 0,68 \\
\hline C26 & $\begin{array}{l}\text { Contiene modelo de acta de eliminación utilizada por el archivo en el proceso de } \\
\text { expurgo }\end{array}$ & 0,75 \\
\hline \multirow[t]{2}{*}{ C27 } & $\begin{array}{l}\text { Se informa acerca de los criterios que deben seguirse antes del envío de la } \\
\text { documentación al archivo (por ejemplo: Eliminación de documentación de apoyo } \\
\text { informativo) }\end{array}$ & 0,63 \\
\hline & DESCRIPCIÓN & \\
\hline \multirow[t]{2}{*}{ C28 } & $\begin{array}{l}\text { Se representan las unidades de descripción, el contexto y el sistema que los ha } \\
\text { producido (ISAD (G)) }\end{array}$ & 0,85 \\
\hline & INSTRUMENTOS DE DESCRIPCIÓN & \\
\hline C29 & Dispone de guía & 0,88 \\
\hline $\mathbf{C} 30$ & Dispone de inventario & 0,53 \\
\hline C31 & Dispone de catálogo & 0,60 \\
\hline \multirow[t]{2}{*}{ C32 } & Contiene otros instrumentos auxiliares & 0,55 \\
\hline & INFORMACIÓN SOBRE FONDOS & \\
\hline C33 & Informa sobre los metros lineales de documentación o unidades de instalación & 0,50 \\
\hline \multirow[t]{2}{*}{ C34 } & Permite la visualización del cuadro de clasificación a través de la web & 0,49 \\
\hline & ORGANIZACIÓN & \\
\hline \multirow[t]{2}{*}{ C35 } & Se hace mención a la fase de organización del archivo, explicando el proceso & 0,43 \\
\hline & ACCESO A LA INFORMACIÓN: PRÉSTAMO Y CONSULTA & \\
\hline C36 & Se recoge el Préstamo externo como servicio del archivo para exposiciones, etc & 0,74 \\
\hline C37 & Se recoge el Préstamo interno como servicio del archivo & 0,86 \\
\hline C38 & $\begin{array}{l}\text { Aparecen reflejadas ciertas formalidades de control, como la normativa de } \\
\text { préstamo de documentos }\end{array}$ & 0,82 \\
\hline C39 & Contiene modelo de solicitud de préstamo o consulta & 0,87 \\
\hline C40 & Indica las instrucciones para cumplimentar el modelo de solicitud de préstamo & 0,80 \\
\hline C41 & Se recoge la Consulta presencial, directa o en Sala como servicio del archivo & 0,59 \\
\hline C42 & Se especifica el horario de consulta de la sala de investigadores & 0,70 \\
\hline C43 & $\begin{array}{l}\text { Se ofrece servicio de consulta de documentos a distancia (Consulta por internet, } \\
\text { correo electrónico, fax, etc) }\end{array}$ & 0,95 \\
\hline \multirow[t]{2}{*}{$\mathrm{C} 44$} & Su fondo se encuentra digitalizado & 0,70 \\
\hline & INGRESO DE FONDOS & \\
\hline $\mathrm{C} 45$ & Contiene instrucciones sobre cómo realizar una transferencia & 0,62 \\
\hline C46 & Aparece el modelo de relación de entrega & 0,75 \\
\hline C47 & $\begin{array}{l}\text { Se refleja un calendario de transferencias o, en su defecto, una previsión de } \\
\text { futuras transferencias al archivo }\end{array}$ & 0,84 \\
\hline
\end{tabular}




\begin{tabular}{|c|c|c|}
\hline & PROYECTOS EN ARCHIVOS & \\
\hline \multirow[t]{2}{*}{ C48 } & Se reflejan los proyectos en los que se encuentra inmerso & 0,83 \\
\hline & NUEVAS TECNOLOGÍAS EN ARCHIVOS & \\
\hline \multirow[t]{2}{*}{ C49 } & Posee algún catálogo que permita visualizar el fondo del archivo & 0,81 \\
\hline & CERTIFICACIONES DE CALIDAD & \\
\hline \multirow[t]{2}{*}{ C50 } & Contiene certificaciones de calidad por alguna entidad certificadora & 0,70 \\
\hline & INDICADORES DE GESTIÓN EN ARCHIVOS & \\
\hline C51 & Se refleja el porcentaje de series identificadas y valoradas en un año & 0,92 \\
\hline C52 & $\begin{array}{l}\text { Aparece reflejado el porcentaje de ocupación de depósitos (en la web o en } \\
\text { memorias anuales) }\end{array}$ & 1,00 \\
\hline C53 & $\begin{array}{l}\text { Contempla el porcentaje de crecimiento anual del volumen de documentación } \\
\text { (en la web o en memorias anuales) }\end{array}$ & 0,86 \\
\hline \multirow[t]{2}{*}{ C54 } & $\begin{array}{l}\begin{array}{l}\text { Se refleja el número de salidas temporales (\% de préstamos) (en la web o en } \\
\text { memorias anuales) }\end{array} \\
\end{array}$ & 0,84 \\
\hline & INSTRUMENTOS DE MEJORA EN ARCHIVOS & \\
\hline C55 & Ofrece el servicio de Reproducción de documentos & 0,69 \\
\hline C56 & $\begin{array}{l}\text { Se miden o cuantifican las consultas realizadas en red (en la web o en memorias } \\
\text { anuales) }\end{array}$ & 0,66 \\
\hline \multirow[t]{2}{*}{ C57 } & Aparecen encuestas de satisfacción o buzón de sugerencias & 0,50 \\
\hline & INSTRUMENTOS DE CONTACTO EN ARCHIVOS & \\
\hline C58 & Se recoge el directorio de personal del archivo & 0,51 \\
\hline \multirow[t]{2}{*}{ C59 } & Se pueden visualizar datos de contacto & 0,88 \\
\hline & LEGISLACIÓN & \\
\hline $\mathbf{C 6 0}$ & Hace referencia a la legislación nacional vigente & 0,88 \\
\hline C61 & Hace referencia a la legislación autonómica vigente & 0,61 \\
\hline \multirow[t]{2}{*}{ C62 } & $\begin{array}{l}\text { Hace referencia a la legislación propia del Ministerio u organismo al que } \\
\text { pertenece) }\end{array}$ & 0,41 \\
\hline & DIFUSIÓN EN ARCHIVOS & \\
\hline C63 & Contiene producción científica que hace referencia al archivo en cuestión & 0,42 \\
\hline C64 & Aparece recogida la agenda cultural del archivo & 0,42 \\
\hline C65 & Existe acceso a exposiciones (virtuales ) & 0,40 \\
\hline C66 & $\begin{array}{l}\text { Recoge Documentos Técnicos en el área de la Archivística (por ejemplo: De } \\
\text { mesas o grupos de trabajo) }\end{array}$ & 0,72 \\
\hline C67 & Se ofertan cursos del área de Archivos & 0,82 \\
\hline C68 & Contiene "Pieza del mes" & 0,77 \\
\hline C69 & Contiene novedades sobre archivos & 0,94 \\
\hline
\end{tabular}




\begin{tabular}{|c|c|c|}
\hline & ENLACES & \\
\hline C70 & Posee enlaces a asociaciones profesionales & 0,75 \\
\hline C71 & Contiene enlaces a normas Archivísticas & 0,78 \\
\hline C72 & Posee otros enlaces de interés & 0,60 \\
\hline \multirow[t]{2}{*}{ C73 } & Posee contador de usuarios o visitas & 0,67 \\
\hline & INFORMACIÓN SOBRE PROCEDIMIENTOS & \\
\hline C74 & $\begin{array}{l}\text { Contiene el manual de normas y procedimientos del archivo, o reglamento del } \\
\text { archivo }\end{array}$ & 0,69 \\
\hline C75 & Aparece el organigrama de la institución a la que pertenece el archivo & 0,72 \\
\hline \multirow[t]{2}{*}{ C76 } & El archivo está incluido en el organigrama de la entidad & 0,73 \\
\hline & INFORMACIÓN DE APOYO & \\
\hline C77 & Contiene glosario de términos & 0,43 \\
\hline C78 & Muestra diagramas sobre procesos o tareas propias del archivo & 0,61 \\
\hline \multirow[t]{2}{*}{ C79 } & Contiene carta de servicios & 0,60 \\
\hline & REDES SOCIALES & \\
\hline C80 & Aparece enlace en el que se remite a su página en Redes Sociales & 0,63 \\
\hline \multicolumn{3}{|c|}{ EVALUACIÓN AUTOMÁTICA } \\
\hline & CARACTERÍSTICAS NEGATIVAS & PESOS \\
\hline C81 & Enlaces Internos Erróneos & $-0,83$ \\
\hline C82 & Enlaces Externos Erróneos & $-0,71$ \\
\hline C83 & Anclajes Erróneos & $-0,26$ \\
\hline C84 & Páginas sin Título & $-0,50$ \\
\hline C85 & Páginas Lentas & $-0,58$ \\
\hline C86 & Páginas sin Atributos de Imagen & $-0,58$ \\
\hline C87 & Páginas Antiguas & $-0,47$ \\
\hline \multirow[t]{2}{*}{ C88 } & Páginas Profundas & $-0,40$ \\
\hline & CARACTERÍSTICAS POSITIVAS & \\
\hline C89 & Páginas Actualizadas & 0,79 \\
\hline C90 & Páginas Pequeñas & 0,55 \\
\hline
\end{tabular}

Fuente: Elaboración propia (2016) 


\section{b) Grado de cumplimiento de cada característica en cada web archivística}

Una vez establecido el peso de cada característica hay que comprobar su grado de cumplimiento en la web de cada Archivo Nacional. En el caso de la evaluación heurística, durante el primer semestre del año 2014, se procede a valorar personalmente el grado de cumplimiento de cada característica en cada sitio web, escogiendo la ponderación 0 (cuando no existe la característica en el Archivo analizado) y 0,$25 ; 0,5 ; 0,75$ o 1 (cuando el grado de cumplimiento de la característica se ha cumplido de menor a mayor grado de éxito, atendiendo a una tabla pormenorizada diseñada por los analistas). Dado que para descubrir heurísticamente el cumplimiento de la característica en el sitio web es imposible profundizar en todos los niveles de la misma, se escogió una muestra representativa de la población que fue analizada detalladamente para comprobar el nivel de profundidad en el que se encontraba cada característica. Así, para la evaluación heurística, además de recoger el grado de cumplimiento de cada característica en la web del Archivo, también se han tenido en cuenta los niveles de profundidad, con el fin de saber cuántos “clics” se requieren para llegar a la característica que se está buscando en la web, tomando como nivel de menor profundidad el 1 y el de más profundidad el 4. Una vez obtenido, tanto el grado de cumplimiento de la característica en el sitio web archivística, como el nivel en el que se encuentra dentro de ella, se ha dividido dicho grado de cumplimiento por el nivel, obteniéndose con ello un valor relativo con el que se ha trabajado para obtener los resultados de la evaluación heurística. Con ello, se ha intentado conseguir un grado de cumplimiento más objetivo, puesto que, con este método, no se valora igual que la característica se encuentre en la página de inicio del Archivo, que en niveles más profundos dentro del sitio web.

En cuanto a la evaluación automática, durante el segundo semestre del año 2014, Linkbot recorre todas las web archivísticas del análisis. En este caso, el grado de cumplimiento de cada característica en cada archivo es el valor extraído automáticamente por Linkbot en su recorrido por el sitio web. También se ha calculado el número de páginas de cada sitio web del Archivo (con Linkbot) y se ha divido el valor de cada característica extraída entre el número de páginas, a fin de hacer los resultados de la evaluación automática más objetivos.

\section{c) Modelo para la calcular los Rankings de los sitios web de Archivos}

Para calcular el peso de los rankings hallados (tanto en la evaluación heurística, como en la automática) y, por consiguiente, la posición que los Archivos Nacionales obtienen en dicha clasificación, se ha puesto en relación la ponderación asignada a cada característica con su grado de cumplimiento en cada web archivística, según la fórmula:

$$
S_{p}=\sum_{i=1}^{n} p_{i} x_{i}
$$


Donde:

- Spes la Suma ponderada de las características de cada Archivo web:

- $\mathrm{p}_{\mathrm{i}}$ es el peso asignado a cada característica i-ésima

- $\mathrm{x}_{\mathrm{i}}$ es el grado de cumplimiento de cada característica i-ésima en cada Archivo web

- $\mathrm{n}$ se corresponde con el número total de características analizadas en cada Archivo Web $=90$ (80 heurísticas y 10 automáticas).

Una vez establecido el procedimiento para calcular los rankings de los sitios web de los Archivos Nacionales en Iberoamérica tras la implementación de modelos ponderados de características subjetivas (ranking heurístico) y automáticas (ranking automático), se pone en relación cada uno de los rankings con los siguientes indicadores:

- Económico: PIB per cápita (2014) (datos en US\$ a precios actuales). Fuente: TheWorldFactbook de Agencia Central de Inteligencia (https://www.cia.gov/library/publications/the-world-factbook/).

- Tecnológico: Porcentaje de la Población que usa Internet (2013). Fuente: Unión Internacional de Telecomunicaciones, Naciones Unidas (http://www.itu.int/es/Pages/default.aspx).

- Demográfico: Población (2013) (en millones). Fuente: Banco Mundial (http://www.bancomundial.org/)

- Cultural: Gasto público en cultura (2013) (en millones de dólares). Fuente: Comisión Económica para América Latina y El Caribe (CEPAL) (http://www.cepal.org/es).

Finalmente, se procede a calcular la correlación entre el ranking obtenido mediante la evaluación heurística y el ranking obtenido mediante la evaluación automática. Para calcular las correlaciones se aplica el Coeficiente de Correlación de Pearson. Aunque existen diversas clasificaciones para establecer el umbral de la correlación, en este trabajo se toma como referencia al autor Ferreiro Aláez (1993), quien considera que la correlación pueden ser: muy fuerte (superior a 0,700), sustancial (entre 0,500 y 0,699) o moderada (entre 0,300 y 0,499).

\section{Resultados y discusión}

Los resultados se agrupan en tres ámbitos geográficos: España, Portugal y Latinoamérica. En el caso de este último, cada resultado es el promedio hallado en el conjunto de los 19 Archivos Nacionales de los países de Latinoamérica.

En primer lugar, se analiza el grado de cumplimiento de las características en el conjunto de los 21 Archivos Nacionales. Con respecto a la evaluación heurística, las "características generales" tienen un promedio de cumplimiento elevado en los 
tres ámbitos analizados, ya que se aproxima al valor máximo (1): España $(0,838)$, Portugal $(0,8)$ y Latinoamérica $(0,718)$ (Anexo I). En cambio, en la evaluación heurística de las "características específicas" el promedio de cumplimiento desciende considerablemente: España $(0,3)$, Portugal $(0,333)$ y Latinoamérica $(0,153)$ (Anexo II). En el caso de la evaluación automática, los promedios de cumplimiento son bajos en los tres ámbitos: España $(0,143)$; Portugal $(0,240)$ y Latinoamérica $(0,174)$ (Anexo III), lo que resulta favorable, puesto que de las 10 características automáticas analizadas, 8 son de carácter negativo. En segundo lugar, se muestran los rankings obtenidos tras aplicar la metodología descrita. Los resultados muestran los Archivos Nacionales clasificados en cuatro Zonas dependiendo de la posición que cada Archivo adquiere en el ranking respectivo. En la Zona 1 se posicionan aquellos que han obtenido los mejores puestos en la clasificación, es decir, los que mayor calidad presentan, a tenor de las características cumplidas, y en la Zona 4 se sitúan los sitios web de Archivos Nacionales con las menores puntuaciones. Además, se compara la situación de cada Archivo en su Zona correspondiente con cuatro indicadores que reflejan el nivel económico, tecnológico, demográfico y cultural del país al que pertenece el Archivo para comprobar el grado de relación de estas variables con los Ranking de calidad de los sitios web.

En la Tabla 3, ordenada descendentemente por el peso de los Archivos,se refleja el ranking de los sitios web de Archivos Nacionales Iberoamericanos teniendo en cuenta los pesos obtenidos mediante la evaluación heurística y evaluación automática. En esta tabla, pueden observarse cuatro zonas (Z1, Z2, Z3 y Z4): dentro de la Zona 1, encontramos los sitios web de Archivos Nacionales que se hallan en las mejores posiciones de la distribución, en el caso de la evaluación heurística, encontramos a: Portugal, Chile, Colombia, Brasil y Ecuador, en el caso de la evaluación automática encontramos a: Puerto Rico, República Dominicana, Nicaragua, El Salvador y México. Por el contrario, en la Zona 4, encontramos en la evaluación heurística, los Archivos Nacionales de: Nicaragua, El Salvador, Panamá, República Dominicana y Puerto Rico. En el caso de la evaluación automática, encontramos a los Archivos Nacionales de: Ecuador, Portugal, Venezuela, Brasil, España y Panamá.

Tabla 3. Evaluación heurística y evaluación automática: ranking web de Archivos Nacionales Iberoamericanos

\begin{tabular}{|c|c|}
\hline \multicolumn{2}{|c|}{ RANKING HEURÍSTICO } \\
\hline PAÍs & PESO ARCHIVOS \\
\hline \multicolumn{2}{|c|}{ Zona 1 } \\
\hline Portugal & 34,000 \\
\hline Chile & 29,000 \\
\hline Colombia & 27,000 \\
\hline Brasil & 25,000 \\
\hline
\end{tabular}




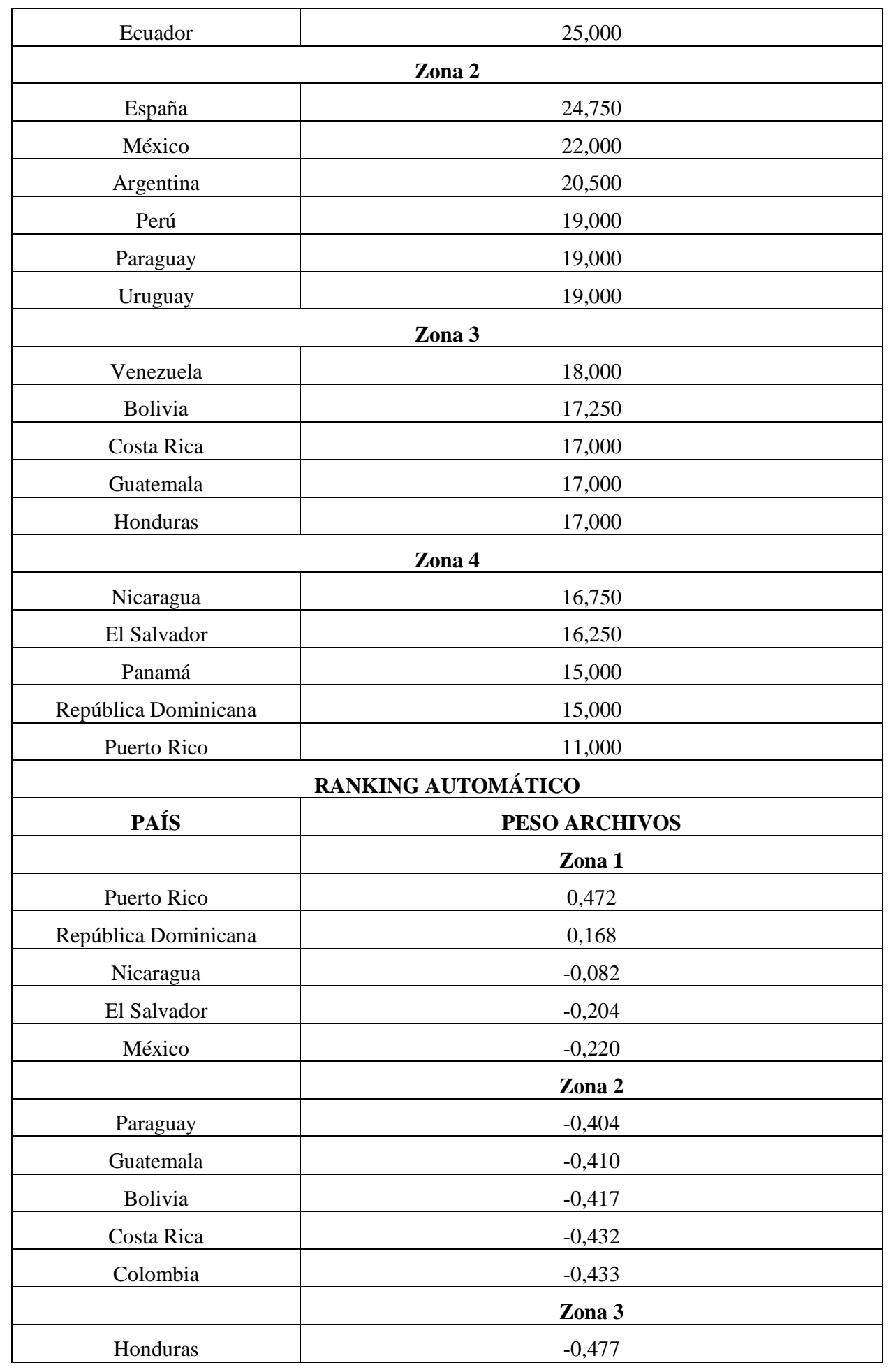




\begin{tabular}{|c|c|}
\hline Uruguay & $-0,515$ \\
\hline Perú & $-0,580$ \\
\hline Chile & $-0,598$ \\
\hline Argentina & $-0,629$ \\
\hline \multicolumn{2}{|c|}{} \\
\hline Ecuador & Zona 4 \\
\hline Portugal & $-0,773$ \\
\hline Venezuela & $-0,802$ \\
\hline Brasil & $-0,949$ \\
\hline España & $-1,323$ \\
\hline Paraguay & $-1,437$ \\
\hline
\end{tabular}

Fuente: Elaboración propia (2016)

Al comprobar el grado de relación que existe entre las posiciones de los Archivos en los rankings y los diferentes indicadores del país al que pertenecen (Tablas 4-ordenada alfabéticamente por el País- y 5), se observa, con respecto a la evaluación heurística, que todas las correlaciones son positivas: sustancial en el caso del Uso de Internet $(\mathrm{r}=0,61)$ y moderada en el resto: Gasto público cultural $(\mathrm{r}=0,35)$, Población $(\mathrm{r}=0,33)$ y PIB per cápita $(\mathrm{r}=0,3)$. Es decir, en general, existe una relación directa (sustancial y moderada) entre la calidad de los sitios web de los Archivos Nacionales y los indicadores del país al que pertenecen, siendo más estrecha en el caso del Uso de Internet, así, cuanto más elevado es el valor de este indicador en el país, mejor posición obtiene su Archivo Nacional en el ranking heurístico implementado, lo que resulta lógico.

En cuanto a la evaluación automática, todas las correlaciones son negativas o inversas: sustancial en el caso del Gasto público cultural ( $\mathrm{r}=-0,51)$, moderada en el Uso de Internet $(\mathrm{r}=-0,46)$ y en la Población $(\mathrm{r}=-0,31)$ y baja en el caso del PIB per cápita $(\mathrm{r}=0,18)$. Es decir, existe relación inversa entre la calidad de los sitios web de los Archivos Nacionales y los indicadores del país al que pertenecen, siendo sustancial en Gasto en Cultura, así, cuanto más elevado es el valor de este indicador en el país, peor posición obtiene su Archivo Nacional en el ranking automático elaborado, lo que resulta paradójico. 
Tabla 4. Rankings e indicadores

\begin{tabular}{|c|c|c|c|c|c|c|c|c|}
\hline \multirow{3}{*}{$\begin{array}{r}\text { País } \\
\\
\text { Argentina } \\
\end{array}$} & \multicolumn{4}{|c|}{ INDICADORES } & \multicolumn{4}{|c|}{ RANKING } \\
\hline & \multirow{2}{*}{$\begin{array}{c}\begin{array}{c}\text { PIB } \\
\text { per } \\
\text { cápita } \\
(\$)\end{array} \\
(\mathbf{2 0 1 4}) \\
12873 \\
\end{array}$} & \multirow{2}{*}{$\begin{array}{c}\text { Uso } \\
\text { Internet } \\
\mathbf{( \% )} \\
(\mathbf{2 0 1 3}) \\
59,9 \\
\end{array}$} & \multirow{2}{*}{$\begin{array}{c}\begin{array}{c}\text { Población } \\
\text { (millones) } \\
\text { (2013) }\end{array} \\
41446246 \\
\end{array}$} & \multirow{2}{*}{$\begin{array}{c}\begin{array}{c}\text { Gasto } \\
\text { Cultura } \\
\text { (millones \$) } \\
(\mathbf{2 0 1 3})\end{array} \\
170,2 \\
\end{array}$} & \multicolumn{2}{|c|}{ Heurístico } & \multicolumn{2}{|c|}{ Automático } \\
\hline & & & & & 20,5 & Zona 2 & $-0,63$ & Zona 3 \\
\hline Bolivia & 3061 & 39,5 & 10671200 & 23,5 & 17,25 & Zona 3 & $-0,42$ & Zona 2 \\
\hline Brasil & 11604 & 51,6 & 200361925 & 2027,7 & 25 & Zona 1 & $-1,32$ & Zona 4 \\
\hline Chile & 14477 & 66,5 & 17619708 & 133,7 & 29 & Zona 1 & $-0,60$ & Zona 3 \\
\hline Colombia & 8762 & 51,7 & 48321405 & 126 & 27 & Zona 1 & $-0,43$ & Zona 2 \\
\hline Costa Rica & 10083 & 45,96 & 4872000 & 68,7 & 17 & Zona 3 & $-0,43$ & Zona 2 \\
\hline Ecuador & 6286 & 40,35 & 15737878 & 79,1 & 25 & Zona 1 & $-0,77$ & Zona 4 \\
\hline El Salvador & 3988 & 23,11 & 6340454 & 14,7 & 16,25 & Zona 4 & $-0,20$ & Zona 1 \\
\hline España & 30278 & 71,57 & 46617825 & 6581 & 24,75 & Zona 2 & $-1,44$ & Zona 4 \\
\hline Guatemala & 3807 & 19,7 & 15468203 & 20,5 & 17 & Zona 3 & $-0,41$ & Zona 2 \\
\hline Honduras & 2361 & 17,8 & 8097688 & 3,9 & 17 & Zona 3 & $-0,48$ & Zona 3 \\
\hline México & 10715 & 43,46 & 122332399 & 1069,6 & 22 & Zona 2 & $-0,22$ & Zona 1 \\
\hline Nicaragua & 1881 & 15,5 & 6080478 & 4,1 & 16,75 & Zona 4 & $-0,08$ & Zona 1 \\
\hline Panamá & 11147 & 42,9 & 3864170 & 25 & 15 & Zona 4 & $-1,70$ & Zona 4 \\
\hline Paraguay & 4305 & 36,9 & 6802000 & 3,2 & 19 & Zona 2 & $-0,40$ & Zona 2 \\
\hline Perú & 8076 & 39,2 & 30375603 & 115,4 & 19 & Zona 2 & $-0,58$ & Zona 3 \\
\hline Portugal & 23481 & 62,1 & 10457295 & 1078,9 & 34 & Zona 1 & $-0,80$ & Zona 4 \\
\hline Puerto Rico & 28529 & - & 3596000 & - & 11 & Zona 4 & 0,47 & Zona 1 \\
\hline $\begin{array}{l}\text { R. } \\
\text { Dominicana }\end{array}$ & 6044 & 45,9 & 10403761 & 35,3 & 15 & Zona 4 & 0,17 & Zona 1 \\
\hline Uruguay & 16199 & 58,1 & 3407062 & 29,4 & 19,000 & Zona 2 & $-0,52$ & Zona 3 \\
\hline Venezuela & 6757 & 54,9 & 30405207 & 115,8 & 18,000 & Zona 3 & $-0,95$ & Zona 4 \\
\hline
\end{tabular}

Fuente: Elaboración propia (2016) 
Tabla 5 Relación rankings-indicadores

\begin{tabular}{|l|c|c|}
\hline \multicolumn{1}{|c|}{ Correlación Pearson (r) } & Ranking heurístico & Ranking automático \\
\hline PIB per cápita (\$) & 0,30 & $-0,18$ \\
\hline Uso Internet (\%) & 0,61 & $-0,46$ \\
\hline Población (millones) & 0,33 & $-0,31$ \\
\hline Gasto Cultura (millones \$) & 0,35 & $-0,51$ \\
\hline
\end{tabular}

Fuente: Elaboración propia (2016)

Finalmente, se aplica el Coeficiente de Correlación de Pearson para comprobar la relación existente entre el ranking web de Archivos Nacionales Iberoamericanos obtenido mediante la evaluación heurística, y el ranking web obtenido mediante la evaluación automática. La correlación obtenida es $\mathrm{r}=-0,403$, es decir, existe una relación moderada negativa o inversa entre los pesos hallados en ambos rankings, lo que significa que, de forma moderada, conforme aumentan unos pesos, descienden otros. Por ello, algunos de los Archivos Nacionales hallados en buenas posiciones en el ranking de evaluación heurística (Zona 1),se sitúan entre las peores posiciones en el ranking de evaluación automática (Zona 4) (como es el caso de Portugal, Brasil o Ecuador), y viceversa, es decir, algunos de los Archivos Nacionales posicionados entre las peores posiciones en evaluación heurística (Zona 4), aparecen situados entre las mejores en evaluación automática (Zona 1) (es el caso de Nicaragua, El Salvador, República Dominicana y Puerto Rico).

\section{Conclusiones}

Con respecto a las características analizadas:

- En evaluación heurística o evaluación por expertos, se ha comprobado en los ámbitos geográficos analizados (España, Portugal y Latinoamérica) que los Archivos Nacionales cumplen mejor las características generales (aquellas que pueden formar parte de cualquier tipo de sitio web), que las de carácter específico (aquellas propias de un sitio web de archivos), lo que resulta negativo, ya que se demuestra que los sitios web de los Archivos Nacionales objeto de estudio no están suficientemente dotados de características concretas propias de una web de archivos, siendo necesario realizar sitios web más orientados al Archivo.

- En relación a las características analizadas mediante evaluación automática, se ha obtenido un grado de cumplimiento bajo en las tres zonas geográficas, lo que resulta positivo e indica que los sitios web de los Archivos Nacionales analizados tienen pocos enlaces erróneos o páginas desactualizadas. 
En cuanto a los rankings obtenidos:

- Respecto a la calidad de los sitios web de los Archivos Nacionales y su relación con los indicadores que miden la Economía, la Tecnología, la Demografía y la Cultura del país en el que se halla el Archivo, se observa:

oEn el ranking de evaluación heurística, que todas las relaciones son sustanciales y moderadas (en especial, en el caso del uso de Internet) de carácter positivo, es decir, conforme mejora el indicador, más favorable es la posición del Archivo en el ranking.

oSin embargo, en el ranking de evaluación automática, aunque también casi todas las correlaciones son sustanciales y moderadas (sobre todo, en el caso del Gasto en Cultura) tienen un carácter negativo o inverso, es decir, conforme mejora el indicador, más desfavorable es la posición del Archivo en el ranking.

oPor ello, se concluye que, dependiendo de las características analizadas y del método de evaluación usado, la Economía, Tecnología, Demografía o Cultura del país en el que se ubica el Archivo Nacional tendrá una relación directa o inversa en la calidad de sus sitios web. Así, por ejemplo, los Archivos Nacionales de Portugal o Brasil (ambos países con un elevado Uso de Internet o Gasto en cultura) tienen sitios web de gran calidad en relación con los objetivos, estructura, diseño, funciones, inventario, consulta, catálogo, novedades, etc. del Archivo (por ello, se posicionan en la Zona 1 del ranking heurístico) pero, sin embargo, su calidad es menor en relación al número de enlaces externos erróneos, de páginas lentas o profundas (por ello, se posicionan en la Zona 4 del ranking automático).

- Respecto a la relación entrelos rankings de evaluación heurística y automática, se evidencia que la correlación es moderada de carácter negativo o inverso $(\mathrm{r}=-0,403)$, lo que significa que, de forma no sustancial, conforme aumentan unos pesos, descienden otros, por lo que Archivos bien posicionados (Zona1) en evaluación heurística, obtienen malas posiciones en evaluación automática (Zona4), y viceversa. Ello corrobora la hipótesis de partida "En general, dado que la evaluación heurística se centra en gran medida en el criterio del analista y la evaluación automática utiliza herramientas informáticas, se prevé que exista una escasa relación entre los rankings obtenidos en ambas evaluaciones”. Es preciso recordar que la evaluación heurística destaca por su carácter subjetivo mientras que la evaluación automática contempla la parte objetiva del estudio, y precisamente este estudio permite concluir que es necesario combinar ambos tipos de evaluaciones para obtener resultados más certeros. 


\section{Referencias bibliográficas}

Acuña, MJ; Agenjo, X (2005).Los archivos en la era digital: el problema (y la solución) de los recursos electrónicos. El profesional de la información, 6 (14), 407-413.

Al-Duwairi, K; Faba-Pérez, C; Vargas-Quesada, B. (2010). Indicadores Webmétricos de carácter Formal para Evaluar el Posicionamiento de las Universidades: el caso de los Países Árabes. Investigación Bibliotecológica: archivonomía, bibliotecología e información, 52 (24), 145-171.

Caballero-Cortés, L.; Faba-Pérez, C.; Moya-Anegón, F. (2009). Evaluación comparativa de la accesibilidad de los espacios web de las bibliotecas universitarias Españolas y Norteamericanas. Investigación Bibliotecológica: archivonomía, bibliotecología e información, 23 (47), 45-66.

Cerdá, J. (2002). "Desarrollo de sistemas de acceso on-line a fondos de archivo Propuesta metodológica”. En INFO: Congreso Internacional de Información (1-122) La Habana.

Cerdá, J. (2008) Archivos locales en la Web El Futuro en la Red. En Actas de las VIII Jornadas de Archivos Aragoneses (151-172). Huesca: Gobierno de Aragón.

Chaín, C; Más-Bleda, A. (2009). Los archivos nacionales hispanoamericanos y la gestión de información de sus sitios web. Biblios, 35, 1-23.

Chaín, C; García, M. (2009). Los contenidos de las web de los archivos históricos estatales. El ciudadano más cerca de la información. Cuadernos de Documentación multimedia, 20, 5-24.

Chao, H. (2002) Assessing de quality of academic libraries on the Web: the development and testing of criteria. Library \& Information Science Research, 24, 169-194.

Clausen, H. (1999). Evaluation of library Web sites: the Danish case. The Electronic Library, 2 (17), pp. 83-87.

Clyde, L.A. (1996) The library as information provider: the home page. The Electronic Library, 6 (14), 549-558.

Clyde, L.A. (2004). School library Web sites: 1996-2002. The Electronic Library, 22 (2), 158-167.

Conde, M.L. (1995). Manual de tratamiento de archivos administrativos. Madrid: Dirección de Archivos Estatales.

Craven, J. (2000). Electronic Access for All: Awareness in Creating Accessible Web Sites for the University Library. Disability and InformationSystems in HigherEducation.

Crespo, S. (2007). Archivos Histórico Provinciales en la red: análisis y evaluación” Boletín de la ANABAD, 2 (1), 497-512.

Cruz, J.R. (1994). Manual de Archivística Madrid: Fundación Germán Sánchez Ruipérez.

Cuadrado, O.; Gómez, D. (2002). Los archivos municipales españoles en línea: evaluación del contenido de sus webs En Actas del IV Jornadas de Archivos Electrónicos, Priego de Córdoba.

Duplá, A. (1997). Manual de archivos de oficina para gestores Madrid: Consejería de Educación y Cultura de la Comunidad de Madrid.

Faba-Pérez, C.; Guerrero-Bote, V.; Moya-Anegón, F. (2004). Fundamentos y técnicas cibermétricas. Mérida: Sociedad de la Información Junta de Extremadura.

Faba-Pérez, C; Guerrero-Bote, V; Moya-Anegón, F. (2005) Self-Organizing Maps of Web Spaces based in Formal Characteristics. Information Processing \& Management, 2, (41), 331-346.

Falk, H. (1999). Library web site innovations. The Electronic Library, 5 (17), 323-328.

Fernández, P. (1999). Manual de organización de archivos de gestión en las oficinas municipales. Granada: CEMCI. 
Ferreiro, L. (1993). Bibliometría: análisis bivariante Madrid: Espasa.

Fuster, F. (1999). Archivística, archivo, documento de archivo necesidad de clarificar los conceptos. Anales de documentación 2, 103-120.

Gil, P. (2001). Tejiendo Archivos: lo que la WWW puede hacer por un archivo. Biblos, 10 (3). 1-12.

Gil, P. (2003). Panorama de los Archivos Universitarios en la Red: El mapa web de archivos de universidades españolas. Archivos Universitarios e Historia de las Universidades, 153-166.

Hassan, Y.; Martín, F. J. (2003). Guía de Evaluación Heurística de Sitios Web [En línea] No Solo Usabilidad 2 <http://wwwnosolousabilidadcom/articulos/heuristicahtm> [Consulta: 20/07/2017]

Heredia, A. (1993). Archivística general: teoría y práctica Sevilla: Diputación Provincial.

Jiménez, M. Evaluación de sitios web, Revista Española de Documentación Científica, 4 (24), 405-431.

Jiménez, M; Ortiz-Repiso, V. (2007). Evaluación y calidad de sitios web. Gijón: Trea.

Joint, N. (2001). Designing interfaces for distributed electronic collections: the lessons of tradicional librarianship. Libri, 51, 148-156.

Kim, H; Lee, H. (2007). "Digital-age trends and perspectives in Korean university archives” The Electronic Library 3, (27), 426-440.

Liria, L. (2001). Archivos virtuales: el reto del Siglo XXI. Congreso de Archivología del MERCOSUR.

Martínez, O. (2000). El Web del Archivo General de la Universidad Carlos III de Madrid”. Boletín de la ANABAD. 1 (50), 21-44.

Más-Bleda, A. (2010). Análisis de las sedes web de los archivos nacionales de Hispanoamérica: estudio de su evolución y propuestas de mejora. Tesis doctoral, Universidad de Murcia.

Más-Bleda, A.; Chaín, C. (2009). Los usuarios y las webs de los archivos históricos nacionales: el caso del Arquivo Nacional da Torre do Tombo (Portugal). Investigación bibliotecológica, 47 (23), 189-212.

Más-Bleda, A.; Thelwall, M. (2016). "Can alternativeindicatorsovercomelanguagebiases in citationcounts? A comparison of Spanish and UK research”, Scientometrics, 109 (3), 2007-2030.

Más-Bleda, A.; Thelwall, M., Kousha, K.; Aguillo, IF (2014). "Do HighlyCitedResearchersSuccessfully use the Social Web?” Scientometrics, 101 (1), 337-356

Nielsen, J.; Thair, M. (2002). Usabilidad de páginas de inicio: Análisis de 50 sitios web Madrid: Pearson Educación.

Novljan, S.; Žumer, M. (2004). Web pages of Slovenian public libraries: evaluation and guidelines. Journal of Documentation, 1 (60), 62-76.

Osorio, N.L. (2001). Web sites of science-engineering libraries: an analysis of content and desing. Issues in Science and Technology. Librarianship.

Providenti, M. (2004). Library Web Accessibility at Kentucky's 4-year Degree Granting Colleges and Universities. D-Lib Magazine, 9 (10).

Roba, O. (2003). Archivos de datos en línea para ciencias sociales, El profesional de la información, 5 (12), 400-410.

Sanz-Caballero, I.M.; Faba-Pérez, C. (2010). El Factor de Impacto Web de los Archivos Universitarios Latinoamericanos: la fiabilidad de los motores de búsqueda para calcularlo, Investigación Bibliotecológica: Archivonomía, Bibliotecología e Información, 50, 157-181. 
Schmetzke, A. (2005). Web Accessibility at University of Wisconsin Libraries. Wisconsin Association of Academic Librarians (WAAL), Newsletter, 3 (22), 5-9.

Spindler, T. (2002). The Accessibility of Web Pages for Mid-sized College and University Libraries. Reference y User Services Quarterly, 2, (42), 149-154.

Stover, M.; Zink, S. D. (1996). World Wide Web home page design: patterns and anomalies of higher education library home pages. Reference ServicesReview, 7-20.

Torrado, S. (2007). La página web del archivo histórico del Instituto Luce: un instrumento de difusión cinematográfica, Anales de Documentación, (10), 413-428.

Anexo I. Promedios de características generales en Archivos Webs Nacionales Iberoamericanos (evaluación heurística)

\begin{tabular}{|c|c|c|c|}
\hline CÓDIGO & ESPAÑA & PORTUGAL & LATINOAMÉRICA \\
\hline C1 & 1,000 & 1,000 & 1,000 \\
\hline C2 & 0,750 & 1,000 & 0,803 \\
\hline C3 & 1,000 & 1,000 & 0,947 \\
\hline C4 & 1,000 & 1,000 & 0,947 \\
\hline C5 & 1,000 & 1,000 & 1,000 \\
\hline C6 & 1,000 & 1,000 & 1,000 \\
\hline C7 & 1,000 & 1,000 & 0,211 \\
\hline C8 & 0,000 & 1,000 & 0,974 \\
\hline C9 & 1,000 & 1,000 & 0,263 \\
\hline C10 & 0,000 & 0,000 & 0,000 \\
\hline C11 & 1,000 & 0,000 & 1,000 \\
\hline C12 & 1,000 & 1,000 & 1,000 \\
\hline C13 & 1,000 & 1,000 & 1,000 \\
\hline C14 & 1,000 & 1,000 & 0,263 \\
\hline C15 & 0,000 & 1,000 & 0,000 \\
\hline C16 & 1,000 & 0,000 & 0,947 \\
\hline C17 & 1,000 & 1,000 & 0,789 \\
\hline C18 & 1,000 & 1,000 & 0,263 \\
\hline C19 & 1,000 & 0,000 & 1,000 \\
\hline C20 & 1,000 & 1,000 & $\mathbf{0 , 7 1 8}$ \\
\hline & $\mathbf{0 , 8 3 8}$ & $\mathbf{0 , 8 0 0}$ & \\
\hline
\end{tabular}


Anexo II. Promedios de características específicas en Archivos Webs Nacionales Iberoamericanos (evaluación heurística)

\begin{tabular}{|c|c|c|c|}
\hline CÓDIGO & ESPAÑA & PORTUGAL & LATINOAMÉRICA \\
\hline C21 & 1,000 & 1,000 & 0,947 \\
\hline C22 & 0,000 & 0,000 & 0,053 \\
\hline C23 & 0,000 & 0,000 & 0,000 \\
\hline C24 & 0,000 & 0,000 & 0,000 \\
\hline $\mathrm{C25}$ & 0,000 & 0,000 & 0,000 \\
\hline C26 & 0,000 & 0,000 & 0,000 \\
\hline C27 & 0,000 & 0,000 & 0,000 \\
\hline $\mathrm{C} 28$ & 0,000 & 0,000 & 0,105 \\
\hline C29 & 1,000 & 0,000 & 0,053 \\
\hline C30 & 1,000 & 0,000 & 0,263 \\
\hline C31 & 1,000 & 0,000 & 0,158 \\
\hline C32 & 1,000 & 0,000 & 0,158 \\
\hline C33 & 0,000 & 0,000 & 0,053 \\
\hline C34 & 0,000 & 1,000 & 0,158 \\
\hline C35 & 0,000 & 0,000 & 0,053 \\
\hline C36 & 0,000 & 0,000 & 0,000 \\
\hline C37 & 0,000 & 0,000 & 0,000 \\
\hline C38 & 0,000 & 0,000 & 0,000 \\
\hline C39 & 1,000 & 0,000 & 0,000 \\
\hline C40 & 0,000 & 0,000 & 0,000 \\
\hline C41 & 1,000 & 1,000 & 0,789 \\
\hline $\mathrm{C42}$ & 1,000 & 1,000 & 0,474 \\
\hline C43 & 0,000 & 0,000 & 0,105 \\
\hline C44 & 0,000 & 0,000 & 0,105 \\
\hline $\mathrm{C45}$ & 0,000 & 0,000 & 0,053 \\
\hline C46 & 0,000 & 0,000 & 0,000 \\
\hline C47 & 0,000 & 0,000 & 0,000 \\
\hline C48 & 0,000 & 0,000 & 0,211 \\
\hline C49 & 1,000 & 0,000 & 0,211 \\
\hline C50 & 0,000 & 0,000 & 0,053 \\
\hline C51 & 0,000 & 0,000 & 0,000 \\
\hline C52 & 0,000 & 0,000 & 0,000 \\
\hline C53 & 0,000 & 0,000 & 0,000 \\
\hline C54 & 0,000 & 0,000 & 0,000 \\
\hline C55 & 0,000 & 1,000 & 0,368 \\
\hline C56 & 0,000 & 0,000 & 0,000 \\
\hline C57 & 1,000 & 0,000 & 0,000 \\
\hline C58 & 1,000 & 1,000 & 0,421 \\
\hline C59 & 1,000 & 1,000 & 0,474 \\
\hline
\end{tabular}




\begin{tabular}{|l|l|l|l|}
\hline $\mathbf{C 6 0}$ & 1,000 & 1,000 & 0,368 \\
\hline $\mathbf{C 6 1}$ & 0,000 & 1,000 & 0,263 \\
\hline $\mathbf{C 6 2}$ & 0,000 & 1,000 & 0,263 \\
\hline $\mathbf{C 6 3}$ & 0,000 & 0,000 & 0,211 \\
\hline $\mathbf{C 6 4}$ & 0,000 & 1,000 & 0,263 \\
\hline $\mathbf{C 6 5}$ & 1,000 & 1,000 & 0,158 \\
\hline $\mathbf{C 6 6}$ & 0,000 & 1,000 & 0,211 \\
\hline C67 & 0,000 & 1,000 & 0,105 \\
\hline C68 & 0,000 & 1,000 & 0,053 \\
\hline C69 & 1,000 & 1,000 & 0,263 \\
\hline C70 & 0,000 & 0,000 & 0,158 \\
\hline C71 & 0,000 & 1,000 & 0,158 \\
\hline C72 & 1,000 & 1,000 & 0,368 \\
\hline C73 & 0,000 & 0,000 & 0,053 \\
\hline C74 & 0,000 & 0,000 & 0,158 \\
\hline C75 & 1,000 & 1,000 & 0,158 \\
\hline C76 & 1,000 & 1,000 & 0,158 \\
\hline C77 & 0,000 & 0,000 & 0,053 \\
\hline C78 & 0,000 & 0,000 & 0,053 \\
\hline C79 & 0,000 & 0,000 & 0,105 \\
\hline C80 & 0,000 & 0,000 & 0,316 \\
\hline & $\mathbf{0 , 3 0 0}$ & $\mathbf{0 , 3 3 3}$ & $\mathbf{0 , 1 5 3}$ \\
\hline
\end{tabular}

Anexo III. Promedios Archivos Webs Nacionales Iberoamericanos (evaluación automática)

\begin{tabular}{|c|c|c|c|}
\hline CÓDIGO & PORTUGAL & ESPAÑA & LATINOAMÉRICA \\
\hline C81 & 0,095 & 0,109 & 0,056 \\
\hline $\mathbf{C 8 2}$ & 0,571 & 0,815 & 0,030 \\
\hline C83 & 0,000 & 0,000 & 0,009 \\
\hline C84 & 0,000 & 0,000 & 0,098 \\
\hline $\mathbf{C 8 5}$ & 0,095 & 0,597 & 0,518 \\
\hline $\mathbf{C 8 6}$ & 0,000 & 0,765 & 0,517 \\
\hline $\mathbf{C 8 7}$ & 0,000 & 0,059 & 0,089 \\
\hline $\mathbf{C 8 8}$ & 0,667 & 0,000 & 0,058 \\
\hline $\mathbf{C 8 9}$ & 0,000 & 0,059 & 0,328 \\
\hline $\mathbf{C 9 0}$ & 0,000 & 0,000 & 0,041 \\
\hline & $\mathbf{0 , 1 4 3}$ & $\mathbf{0 , 2 4 0}$ & $\mathbf{0 , 1 7 4}$ \\
\hline
\end{tabular}

